\title{
Comparing Cursor Orientations for Mouse, Pointer, and Pen Interaction
}

\author{
Barry A. Po, Brian D. Fisher, and Kellogg S. Booth \\ Department of Computer Science, University of British Columbia \\ 201-2366 Main Mall, Vancouver, B. C., Canada V6T 1 Z4 \\ \{po, fisher, ksbooth\}@cs.ubc.ca
}

\begin{abstract}
Most graphical user interfaces provide visual cursors to facilitate interaction with input devices such as mice, pointers, and pens. These cursors often include directional cues that could influence the stimulus-response compatibility of user input. We conducted a controlled evaluation of four cursor orientations and an orientationneutral cursor in a circular menu selection task. Mouse interaction on a desktop, pointer (i.e. wand) interaction on a large screen, and pen interaction on a Tablet PC were evaluated. Our results suggest that choosing appropriate cursors is especially important for pointer interaction, but may be less important for mice or pens. Cursors oriented toward the lower-right corner of a display yielded the poorest performance overall while orientation-neutral cursors were generally the best. Advantages were found for orientations aligned with the direction of movement. We discuss these results and suggest guidelines for the appropriate use of cursors in various input and display configurations.
\end{abstract}

Categories \& Subject Descriptors: H.1.2. [Models and Principles]: User/Machine Systems - Human factors; H.5.2. [Information Interfaces and Presentation (e.g., HCI)]: User Interfaces - Input devices and strategies (e.g., mouse, touchscreen)

General Terms: Human Factors, Measurement, Theory

Keywords: Cursors, mice, styli, wands, pens, pointing, desktops, large screens, small screens.

\section{INTRODUCTION}

Visual cursors are an integral part of many graphical user interfaces (GUIs). On a typical desktop, cursors are used both as an indicator of system state ("iconic cursors") and as a means of providing visual feedback for user input

Permission to make digital or hard copies of all or part of this work for personal or classroom use is granted without fee provided that copies are not made or distributed for profit or commercial advantage and that copies bear this notice and the full citation on the first page. To copy otherwise, or republish, to post on servers or to redistribute to lists, requires prior specific permission and/or a fee.

CHI 2005, April 2-7, 2005, Portland, Oregon, USA.

Copyright 2005 ACM 1-58113-998-5/05/0004 ...\$5.00.

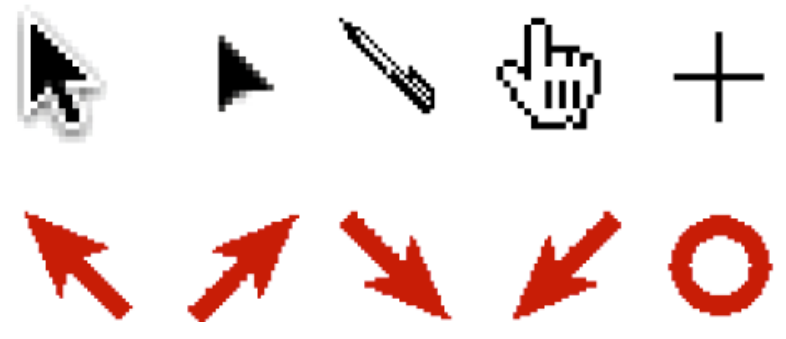

Figure 1. Some common pointing cursors used in GUIs (top row) and the cursors tested in our controlled evaluation (bottom row).

("trackers"). In this latter role, cursors are frequently represented as arrows pointing toward a particular direction on the display. Figure 1 presents some of the more common pointing cursors seen in today's desktop GUIs.

The most popular pointing cursor is an arrow oriented toward the upper-left corner of a display. This cursor is seen with little variation in all of the most popular GUIs and has a history that dates at least as far back as the first Bravo editor prototypes at Xerox PARC in September of 1974. Although the development of the mouse itself is well documented, virtually no published information exists regarding the design and selection of the upper-left arrow cursor used for mouse pointing. According to Butler Lampson, who was one of the primary developers on the Bravo project, the upper-left cursor was chosen both because it matched the Bravo developers' intuition that up and to the left seemed most natural given the orientation of mouse and screen, and because it was one of the few cursor orientations that looked acceptable on the graphics hardware available at the time (personal communication, August 2, 2004). We contacted several other Bravo developers, and none recalled formal user testing of different cursor orientations.

It is interesting then that many GUIs continue to adopt this particular interface convention without substantial evaluation. Different cursor orientations could influence the stimulus-response compatibility of user input and could have a considerable impact on user performance. It remains unclear how different cursors might affect performance 
across a variety of input devices. We were interested in characterizing these performance differences and what they could mean for GUI design.

We present a controlled evaluation of four different arrow cursor orientations (toward the upper-left, upper-right, lower-left, and lower-right of a display), and one orientation-neutral cursor (a circle) in a menu selection task across three different kinds of input devices: mice for desktop interaction, pointers for freehand interaction with large screens (i.e. wand or laser pointer interaction [21]), and pens for interaction with smaller displays. We begin by reviewing existing work related to cursor orientations in the HCI and psychological literature, followed by a description of our hypotheses, experimental methodology and results. We discuss the implications of our results for user interface design and suggest some guidelines for the use of graphical cursors with different kinds of input devices on various displays.

\section{BACKGROUND AND RELATED WORK}

Different cursor orientations may affect user performance because of stimulus-response (S-R) compatibility, a phenomenon that has been widely studied in cognitive psychology and ergonomics $[1,9,15,17,30]$. With respect to visual user interfaces, S-R compatibility can be defined as the degree to which the mapping between the position and orientation of a visual stimulus (such as the arrow direction on a cursor) matches that of the motor movement (such as the location of a target and intended direction of cursor movement). Compatible mappings are believed to facilitate performance while incompatible mappings are believed to interfere with performance.

In HCI, John and Newell have used a GOMS theory of S-R compatibility to model cognitive user performance in recalling computer command abbreviations [14]. Their subsequent work extended this S-R compatibility model to the domain of expert transcription typing [13]. S-R compatibility is also acknowledged as a factor influencing user stress and cognitive load [26].

There is substantial empirical evidence that directional cues can influence S-R compatibility. Broadbent and Gregory found that when participants were asked to respond to a left or right visual stimulus by pressing a left or right key, left responses were faster to left stimuli than to right stimuli while right responses were faster to right stimuli than to left stimuli [1]. Several experiments by Worringham and Beringer have found that physical controls with implicit directional cues, such as levers, joysticks, and rotary knobs, have effects on responses toward particular directions [30, 31]. A subsequent experiment by Chua, Weeks, Ricker, and Poon found similar effects and demonstrated that spatial compatibility could be affected by global spatial relationships, such as participant orientation [7].
Kantowitz, Triggs, and Barnes also found that visual arrows can influence directional compatibility [15].

S-R compatibility is especially important in GUI design because the mapping of visual stimulus to motor response is central to "direct manipulation" metaphors for interaction. Experimental evidence suggests that the effects of S-R compatibility can never be fully "trained away," meaning that even expert GUI users still benefit from compatible mappings between visual stimulus and motor response [29]. Other evidence suggests that it is difficult to judge what the most compatible S-R configurations will be among several possible design alternatives, so it could be easy for designers to make mistakes about what mappings constitute the most S-R compatible configuration in a user interface $[27,29]$.

Very little work has been done to assess the impact of varying cursor orientations, although an experiment by Phillips, Meehan, and Triggs has directly evaluated the impact of cursor orientation on mouse positioning movements for desktop displays [22]. They found that the directional cues provided by a mouse cursor could induce performance differences. However, they found that arrow cursors compatible with the direction of motion led to slower movements and less efficient cursor trajectories, which is an interesting result because it is at odds with the previous experimental literature.

The evaluation we present here is related to Phillips, Meehan, and Triggs's work, but our goals are substantially different and our approach differs from theirs in many key respects. In addition to examining pointing conditions not reported in their work, we studied compatibility effects with input devices other than mice. These differences are fully described in the sections that follow.

\section{Mice, Pointers, and Pens}

Mice are the predominant pointing devices for conventional desktop GUIs. Their performance characteristics have been studied extensively in HCI [3, 19, 20]. However, as large screens and handheld devices become more common, understanding other pointing devices such as pointers and pens is becoming more important.

Comparing the performance of other input devices to mice has been the subject of several studies. Card, Mackinlay, and Robertson have proposed a unified framework for designing and analyzing different input devices [4]. Sears and Shneiderman have suggested design strategies for using touchscreens instead of mice [25]. Myers et al. compared relative performance differences between several input devices, including laser pointers, touchscreens, handhelds, and mice [20]. Po, Fisher, and Booth conducted a comparative study of mouse and touchscreen performance across the upper and lower visual fields of users [23]. 

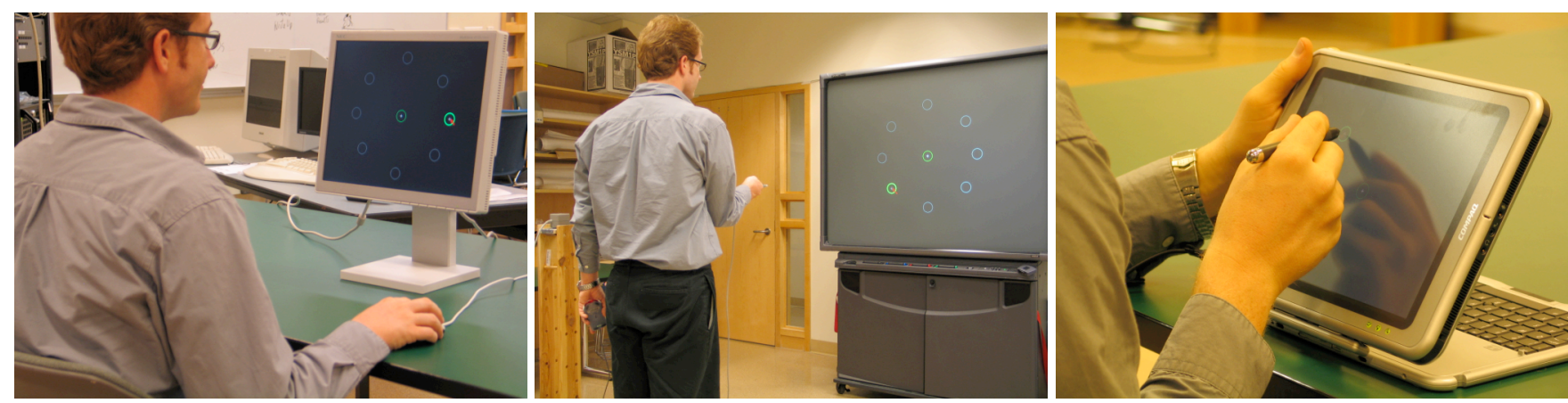

Figure 2. The three input devices compared in our controlled evaluation. From the left: (a) mouse on a desktop, (b) pointer on a large screen, and (c) pen on a Tablet PC.

In our current study, we chose to evaluate cursor orientation effects as they relate to three different kinds of input devices: mice, pointers, and pens. Although it is well established that each of these input devices has different performance characteristics, little work has been done in HCI to compare the impact of S-R compatibility across different input devices. There is evidence to suggest that the compatibility of a particular S-R mapping can be influenced by hand posture and orientation, which could be important in comparing interaction performance across different input devices because mice, pointers, and pens are each held in substantially different ways $[6,17]$.

For the purposes of this study, we define a pointer to be an ungrounded (but not necessarily wireless) input device that allows users to directly point at a display from a distance (see Figure $2 b$ ). The tip of the pointer casts a straight-line ray (like a laser pointer would) onto the display and the intersection of this ray with the display is where the user is pointing and where a visual cursor is rendered. In immersive virtual environments, such pointers are often implemented with a 3D tracking wand or stylus [12]. With recent interest in camera-tracked laser pointer interaction, pointer input is making its way into mainstream interaction as an alternative for interaction with shared displays and other large screen environments [16, 20,28].

The wider availability of tablet displays and handheld devices has made pen input an important area of study. Citrin et al. have investigated the potentials and limitations of pen-based input for personal workstations [8]. Two experiments by Ren and Moriya have characterized the relationship between pen-based input and item selection [24]. Another study by Hancock and Booth has looked at pen-based menu selection across horizontally- and vertically-oriented displays [11].

\section{HYPOTHESES}

Based on previous experimental work, it appears plausible that graphical elements with implicit directional cues could affect the S-R compatibility of user input. This suggests that the directional (oriented) cursors used in many GUIs could have a substantial effect on user movement and positioning within a display. In our current study, we investigated the validity of three specific experimental hypotheses:

1. Graphical cursors indicating direction will show performance differences consistent with those predicted by theories of S-R compatibility, regardless of input method. Specifically, pointing movements with directional cursors aligned with specific axes of movement should yield improved movement times and positioning performance. When directional cursors are not aligned with the direction of movement, user performance will be negatively affected.

2. Orientation-neutral cursors will yield the best performance overall, regardless of input method. While these cursors should not confer any advantages for movement toward any particular directions, they also should not suffer from any performance penalties associated with incompatible mappings. When performance is considered across a variety of movement directions, averaged performance should be better relative to cursors that cue for movement in one specific direction.

3. The degree to which varying cursor orientations will affect movement and positioning performance is dependent on the input method. This is partially because different hand postures are believed to affect S-R compatibility but also because different input devices are known to have unique performance characteristics. Based on previous work, we predict that pointer input will yield the largest effects, mouse input will yield intermediate effects, and pen input will yield the smallest effects from cursors of different orientations.

\section{METHODS}

To test our hypotheses, we conducted a controlled user study consisting of a circular menu selection task with different cursor orientations and input devices. The circular menu task shares similarities to a menu evaluation task conducted by Callahan, Hopkins, Weiser, and Shneiderman [2]. The task also resembles several previous experiments that examined various aspects of S-R compatibility in cognitive psychology $[5,6,10,17]$. 

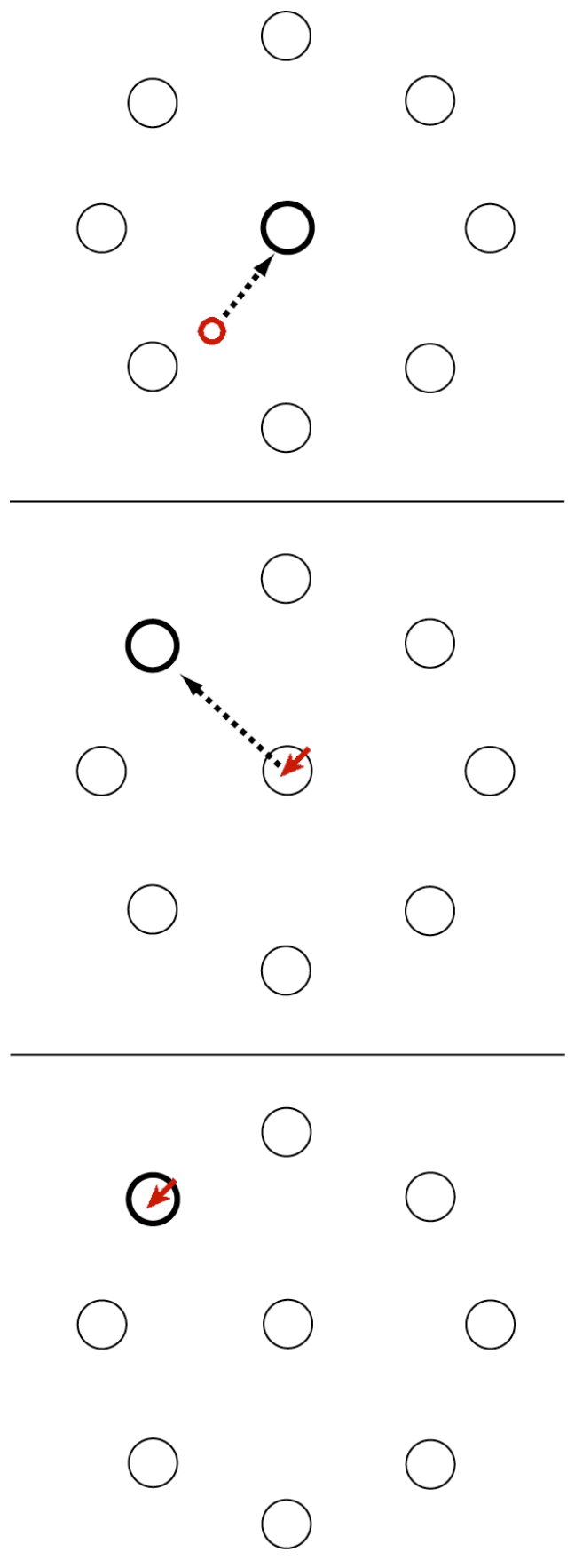

Figure 3. A typical trial in our controlled user study. The dashed arrows indicate direction of movement and were not actually present on the display. Subjects started a trial by selecting a circle located at the center of the display using the orientation-neutral circle cursor. The cursor would change to one of the five cursor representations and one of the eight circular menu positions would change color, indicating where subjects were expected to point. Subjects were instructed to select the center of the highlighted item and to emphasize speed and accuracy equally.
We used a fully counterbalanced, completely withinsubjects experimental design. Subjects were instructed to complete three blocks of 120 menu selection trials. Each block used a different input device to complete a set of trials. Figure 3 presents a visual description of a typical trial. The order in which blocks were presented to subjects was fully counterbalanced. Within each block, trials were fully randomized in a manner consistent with other experiments of this kind, meaning that every block of trials had a unique order of presentation and that every ordering was visibly different from all other orderings given to all subjects.

\section{Subjects}

Twelve subjects participated in the study. Three were male and nine were female. Their ages ranged from 18 to 34 years. To minimize experimental bias due to handedness, we ensured that all of our subjects were right-handed via self-report. We also ensured that all subjects had normal or corrected-to-normal vision via self-report.

\section{Apparatus}

Figure 2 presents the apparatus used in the study. Each block of experimental trials used a different input and display device for menu item selection. A PC workstation was used to render trials in the mouse and pointer trial blocks. A Compaq TC1000 Tablet PC was used to render trials in the pen block. Both machines ran platformappropriate versions of Microsoft Windows XP and used the native mouse pointing drivers provided by the operating system. Movement times were collected using platformnative calls to a high-resolution system timer with a sampling resolution of approximately $1 \mathrm{~ms}$. In all conditions, a constant level of room illumination was maintained.

In the mouse condition, a 17" LCD flat panel display with a resolution of $1024 \times 768$ pixels was used (Figure 2a). A Logitech optical mouse was used as the input device. Mouse gain was set to a hand-display movement ratio of $1: 1$ so that the mouse moved the same distance in hand space as it did in display space. This was done intentionally to allow for a fair comparison of compatibility effects across the different input devices tested.

In the pointer condition, a 66" rear-projection SMART Board 3000i with a resolution of $1024 \times 768$ pixels was used (Figure 2b). The SMART Board was only used as a display; its touch functionality was not used in this experiment. The 3D pointer of an electromagnetic Polhemus Fastrak was held in the right hand of subjects while a push-button mouse was held in their left hand. The Fastrak transmitter and 3D pointer were set up in a space free of metallic interference and the 3D pointer was calibrated and registered before each experiment session.

In the pen condition, a Compaq TC1000 Tablet PC with a resolution of 1024x768 pixels was used (Figure 2c). The Tablet PC was configured to operate in landscape (wide) 
format and the display was inclined at an angle of 45 degrees. The Tablet PC pen used for input was calibrated before each experiment session.

\section{Procedure}

Each subject participated in a single session lasting forty minutes. During a session, an experimenter was present at all times. In the mouse condition, subjects were seated at a distance of $50 \mathrm{~cm}$ from the LCD display. In the pointer condition, subjects stood upright at a distance of $200 \mathrm{~cm}$ from the SMART Board display. In the pen condition, subjects were seated at a distance of $30 \mathrm{~cm}$ from the Tablet PC. Trials in each block consisted of a single pointing movement from the center of a display to one of eight equally distant menu positions with one of five different cursor representations: arrows pointing to the upper-left, upper-right, lower-left, and lower-right of a display, or an orientation-neutral circle (Figure 3). The arrow cursors used in this experiment were oriented at 45-degree angles (Figure 1). Subjects were given five-minute breaks between successive blocks of trials.

All of the visual elements in this study were rendered to subtend the same visual angle across each of the different display and subject viewing distances. All cursors, including the circle cursor, subtended a visual angle of approximately two degrees in all display configurations. Target menu items were eight circles, four degrees of visual angle in size and ten degrees distant from the center of the display. These targets were equally spaced in a circular fashion around the center of the display to form a circular menu. Each combination of trial parameters was repeated three times, yielding a total of 5 cursor orientations $\times 8$ menu positions $\times 3$ input devices $\times 3$ repetitions $=360$ trials per subject. Each trial block consisted of the complete set of 120 trials using one input device.

All visual elements were rendered against a black background. Individual trials within blocks were initiated by having subjects point and select a four-degree circular item at the center of a given display using the orientationneutral circle cursor. This cursor was used to begin all trials because we wanted to avoid any possible interference between arrow cursors used to initiate trials and the cursors used for the pointing movement within a trial. Once the center circle was selected, one of the eight surrounding menu items would be highlighted, indicating where subjects were expected to point. The orientation-neutral cursor would either change into one of the four arrow cursors or remain the same (for trials with an orientation-neutral cursor). Subjects were instructed to point at the center of highlighted items and to emphasize speed and accuracy equally. The display would reset to the beginning of a new trial upon completion of the pointing movement and item selection.

\section{Mouse Condition}

In the block of mouse input trials, subjects were provided with a Logitech optical Wheel Mouse. The mouse was placed on the desktop directly in front of subjects. Subjects pointed at onscreen items by moving the presented visual cursor to graphical elements and then left-clicking the mouse.

\section{Pointer Condition}

In the block of pointer trials, subjects were instructed to hold a Polhemus Fastrak 3D pointer in their right hand while holding a push button mouse in their left hand. Subjects pointed at onscreen items by aiming the 3D pointer directly at graphical elements and clicking a button on the mouse held in their left hand. This two-handed pointer-and-mouse approach allowed us to capture subject data without the errors associated with the "pen-drop" phenomenon common to pointer interaction [20].

\section{Pen Condition}

In the block of pen input trials, subjects were provided with a tablet-sensitive pen for interaction with the Tablet PC. Although the Tablet PC was situated on a table, subjects were asked to hold the Tablet PC with their left hand for extra stability. The pen was held in the right hand and subjects pointed at onscreen items by directly tapping at graphical elements to indicate selection.

\section{Training}

Every subject received a minimum of twenty practice trials prior to each block of experimental trials. Practice trials consisted of trials presented in the same fashion as experimental trials, with randomized cursor orientations and items for selection. Practice trials were presented until both subject and experimenter were satisfied with the subject's ability to complete the task as instructed.

\section{RESULTS}

To determine whether quantitative evidence supported our experimental hypotheses, we looked at primary measures of total movement time and movement precision. Total movement time was defined as the period of time from trial initiation to the point at which a highlighted item was selected, measured in milliseconds (ms). Movement precision was measured in two ways. First, a standard rootmean-square (RMS) index from the center of each menu position was calculated for each pointing movement made in a trial. Second, effective target width was calculated for each menu position and cursor orientation using the standard deviation method, which is often computed when analyzing 2D movement performance using Fitts Law [18].

We used a factorial analysis of variance with repeated measures (ANOVA-RM) over three independent factors of input device, menu position, and cursor type to analyze movement time, RMS index, and effective target width. We conducted an assessment of sphericity for the ANOVA by applying Mauchly's test and by visually observing 


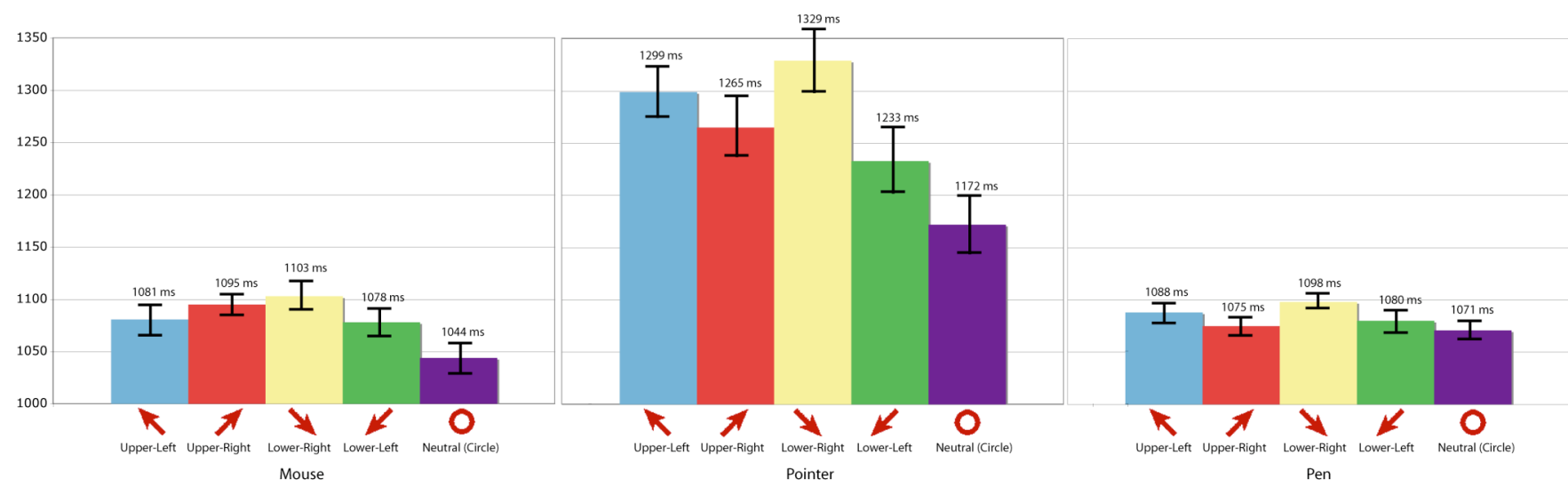

Figure 4. Bar graphs of movement time averaged across the eight menu positions tested in our evaluation. Each bar graph represents the movement times for one input device and each graph is broken down into movement times for each of the cursor types tested. Error bars represent standard errors of the mean. We found that the orientationneutral circle cursor provided the best overall performance while the cursor oriented toward the lower-right was worst overall across all devices. The relative performance differences between cursor types were seen to differ across input devices. Pointer input showed the largest differences while mice and pen input had relatively smaller effects.

collected data. Based on these, we decided it would be prudent to be conservative in our analysis, so we applied a Greenhouse-Geisser correction to all of the relevant statistical analyses.

If cursor orientation had an impact on movement performance, this would be observed in the ANOVA as statistically significant $(p \leq .05)$ two-way interaction effects between menu position and cursor orientation for all three dependent measures. To assess the overall performance of different cursors along all directions of movement, which is important when considering general visual feedback solutions for GUI design, we looked for a corresponding main effect for cursor type and we also calculated averaged movement times, RMS indices, and effective target widths across all eight menu positions. These averaged movement times were subjected to an additional two-way ANOVA across input device and cursor type.

\section{Movement Time}

Our primary repeated measures ANOVA found a statistically significant two-way interaction between menu position and cursor type $[\mathrm{F}(2.46,27.11)=3.88, p=.033]$. This suggests that particular cursor orientations do have an impact on movement time performance toward particular directions of movement. A corresponding two-way interaction between input device and cursor type was also statistically significant $[\mathrm{F}(2.75,30.26)=3.58, p=.028]$. This supports our hypothesis that the impact of different cursor types on movement time can differ across input devices.

We compared performance for those trial situations where cursor type and menu position were compatible (i.e. an upper-left cursor was used to point at the menu item in the upper-left direction and so on) against the averaged movement time for that particular menu position, regardless of cursor type. This comparison was done to measure the effect size difference between compatible mappings and "expected" movement performance. In all of the compatible situations, movement times yielded consistent average improvements of approximately $35 \mathrm{~ms}$ for mouse input (about 3.3\% faster than average), $65 \mathrm{~ms}$ for pointer input (about $5.5 \%$ faster), and $17 \mathrm{~ms}$ for pen input (about $1.5 \%$ faster).

The same comparison was done to measure the effect size differences between incompatible mappings (i.e. when an upper-left cursor was used to point at the menu item in the lower-right direction and so on). In these incompatible situations, movement times again yielded consistent, but relatively smaller, performance penalties. With the incompatible mappings, mouse input was observed to be about $18 \mathrm{~ms}$ slower (1.7\% slower), pointer input was $43 \mathrm{~ms}$ slower (3.7\% slower), and pen input was about $9 \mathrm{~ms}$ slower ( $0.8 \%$ slower) on average.

The ANOVA also revealed a secondary main effect of cursor type, which suggests that simply varying the cursor that was used for movement was enough to cause some change in movement time performance $[\mathrm{F}(2.28,25.07)=$ $5.55, p=.008]$. No other main effects were observed for either input device $[\mathrm{F}(1.71,18.81)=2.10, p=.155]$ or menu position $[\mathrm{F}(3.06,33.67)=2.54, p=.072]$.

Figure 4 presents bar graphs of averaged movement time across all tested movement directions, broken down by cursor orientation for each input device. Our accompanying two-way ANOVA for input device and cursor type for these averaged times yielded an interaction effect between both independent factors $[\mathrm{F}(2.75,30.26)=3.58, p=.028]$. This indicated that some cursor types performed better than others overall and the degree to which these cursors differed also depended on input type. The orientationneutral cursor we tested had the best averaged performance for all input devices. The cursor oriented toward the lower right of the display had the worst averaged performance 
overall for all input devices. This difference was most apparent with pointer input, where movement times differed by $157 \mathrm{~ms}$ between the orientation-neutral cursor and the lower-right cursor. These differences were smaller with mouse and pen input, which demonstrated movement time differences of $59 \mathrm{~ms}$ and $27 \mathrm{~ms}$ respectively.

The orientation-neutral cursor consistently outperformed the upper-left arrow cursor that represents the orientation used in many popular GUI systems. The differences between performance with the upper-left cursor and orientation-neutral cursor was again most apparent with pointer input, where movement times differed by $127 \mathrm{~ms}$. With mouse input, movement times differed between these two cursors by approximately $37 \mathrm{~ms}$. Pen input movement times differed by $17 \mathrm{~ms}$. These differences appear to constitute noticeable improvements in movement time performance, suggesting that there may be practical situations where a neutral-orientation cursor would be beneficial over the standard upper-left cursor usually employed.

As a final analysis of movement time, we averaged subject trials across all eight tested menu positions and all five cursor orientations. This was done to give us another perspective on the performance differences between mouse, pointer, and pen input independent of S-R compatibility effects. We performed a one-way ANOVA across all three input devices and found that movement time performance was significantly different between mice, pointers, and pens $[\mathrm{F}(1.68,18.50)=62.98, p<.001] . \quad$ Average mouse movement times were $1075 \mathrm{~ms}$ in duration, pointer movement times were $1193 \mathrm{~ms}$, and pen movement times were $1081 \mathrm{~ms}$. Thus, we found that the pointer was the most difficult to use, while mice and pen input were relatively similar in performance. These are findings that are consistent with previous research reported in the HCI literature.

\section{Movement Precision}

With respect to movement precision, our primary repeated measures ANOVA determined that there was a statistically significant main effect for input device on measured RMS and effective target width, consistent with findings from other research that has compared the relative performance differences between input devices $[\mathrm{F}(1.20,13.16)=$ $1841.00, p<.001$ for both measures since effective target width is derived from RMS]. Both mouse and pen interaction were observed to have roughly comparable movement precision performance, but pointer input was substantially worse. This could be attributed to the fact that both mouse and pen interaction took place on stable surfaces, while pen input was susceptible to movement effects such as hand jitter.

When compared to the actual target widths of approximately four degrees of visual angle in size, effective target widths remained roughly the same for mouse and pen interaction. However, for pointer interaction, the effective target size was nearly eight times smaller. Because subjects were instructed to emphasize speed and accuracy equally and no speed-accuracy tradeoff was observed in the subject movement data, it is possible that subjects had more difficulty maintaining precise movement with a freehand pointer.

The primary repeated measures ANOVA also found a corresponding significant main effect for cursor type on measured RMS and effective target width $[\mathrm{F}(2.59,28.44)=$ $3.18, p=.045$ for both measures]. Since no other statistically significant interactions or main effects were observed, we were unable to find any potential S-R compatibility effects for positioning and particular mappings of cursor type and movement direction. However, the main effect of cursor type indicates that choice of cursor representation could at least have a small impact on movement precision.

The effect size of cursor type on movement precision is comparatively smaller than the differences across input devices. Effective target widths were computed to be somewhat larger in visual angle when using the orientationneutral circle cursor and upper-right arrow cursor. However, these improvements were relatively small, yielding effective width increases of only 0.3 degrees for the orientation-neutral cursor and 0.15 degrees for the upper-right arrow cursor. All of the other cursor representations yielded size differences of less than 0.1 degrees, which could conceivably be considered not to be large enough to be practical or meaningful. We found similar results with the calculated RMS indices for movement precision, which yielded very small, but consistent differences in favor of the orientation-neutral circle cursor compared to all of the other cursor representations.

\section{Cursor Preferences}

Several study participants reported at the end of their sessions a subjective preference for the orientation-neutral circle cursor compared to all of the others tested, although none of them could give a good clear explanation as to why this was the case. Most of these subjects claimed that the orientation-neutral cursor felt "easier" to use, and some were surprised that this particular cursor was not more common in desktop GUI environments. This particular preference is well supported by the empirical data we collected during the study.

\section{DISCUSSION}

Our results have demonstrated that S-R compatibility is influenced by choice of cursor representation and that these compatibility effects can have a substantial impact on user performance when interacting with GUIs using various input and display configurations.

We had considered an alternative experimental design that blocked trials by cursor orientation instead of input device, which would have yielded five blocks of trials instead of 
three (one block for each cursor). Such a design would have minimized the possibility of "contextual interference" effects as cited in the literature [32]. We decided against this design because we believed it would be too demanding on subjects to move between different input devices across trials within a block. As our results demonstrate, our chosen blocking scheme did not appear to influence subject performance.

The fact that a circular cursor was always used to initiate trials and that no cursor change occurred during orientationneutral trials might explain why the circular cursor was observed to have superior performance. However, this also suggests that cursor changes would interfere with trials where cursors were compatible with movement direction. Despite the change in cursor representation in these trials, our results indicate that movement performance in these specific instances still yielded an advantage relative to postulated incompatible cursors. Thus, there is little evidence that a cursor change in directional cursor trials was a major confound.

Our experimental design differs substantially from the protocol used in the Phillips, Meehan and Triggs study, although our study could be considered to be an extension of their work [22]. Their study focused on determining the presence or absence of S-R compatibility effects with different cursor orientations. We were more interested in characterizing possible compatibility effects for various GUI scenarios, including large screen and handheld displays, and in comparing their relative impact. We were also interested in developing practical guidelines that could be helpful for GUI designers. Thus, pointing movements were restricted to a single dimension in their experiment while our study considered 2D (bivariate) pointing movements. They focused exclusively on mouse positioning while we considered a wider variety of input devices (mice, pointers, and pens). In addition, their experiment only considered three cursor orientations (upper-left, upper-right, and a crosshair cursor) while we tested two other orientations (lower-left and lower-right) and a different orientation-neutral cursor (a circle instead of a crosshair).

The differences in their results and the ones observed in our study could be due to the implicit differences in experimental designs. It is possible that SR-compatibility manifests itself differently in $1 \mathrm{D}$ versus $2 \mathrm{D}$ pointing tasks, or that differences in studied cursor representations were important. It is also conceivable that button pressing, as done in our study but not in theirs, could have altered the experimental task enough to influence compatibility effects.

S-R compatibility and the kinds of visual cues that affect movement responses are important for existing systems and will increase in importance as designers create new graphical interface designs and input devices with unique form factors. Being aware that many elements can influence S-R compatibility, including cursor representation, hand posture, and user orientation, is an incremental step along the way toward understanding the elements that constitute effective GUI designs.

Based on our findings, we present the following guidelines for effective use of visual elements and cursors in GUIs with respect to maintaining S-R compatibility:

1. Where appropriate, consider the use of orientationneutral visual cursors and be careful with the use of visual cursors that include directional cues.

By using a cursor representation free of directional bias, pointing performance could be made more consistent. Our results show that such cursors are likely to improve average movement time performance. This could be most useful in GUIs that employ layouts where interactive elements are located all over the display. It seems reasonable for designers to carefully consider the relative benefits of assuming the use of a directional cursor for general interaction when evidence exists that orientation-neutral cursors might better facilitate user performance.

2. In situations where many interactive elements might be clustered in one particular area of a display (i.e. palettes or toolbars), choosing a cursor tailored to the direction of movement could be helpful.

Our user study found that cursor orientations compatible with the direction of movement facilitated movement performance. Thus, in situations where many interactive elements might be clustered in one particular area of the display (i.e. a palette or toolbar), choosing a cursor tailored to one specific direction might aid users who will be pointing along a particular direction of movement quite frequently. This could be especially relevant for systems where movement time is a critical factor.

3. Where movement precision and positioning is important, consider using an input device that rests on a stable surface or use a touch-based input method, such as pen input.

From our user study, we found that ungrounded pointer input was relatively more difficult to use compared to mouse or pen input, which is a result often reported in the literature. Pen input showed the least susceptibility to S-R compatibility effects from different cursor representations, which could be important under certain circumstances. In some cases, it may be impossible to use other methods of input or it may be preferable to use a pointer (i.e. in a classroom or shared display setting), in which case it is important to ensure that GUI elements provide the most S$\mathrm{R}$ compatible situation possible to users.

4. Do not assume that it is easy to design a user interface that has $S$ - $R$ compatible mappings and be aware of visual elements that could inadvertently affect compatibility mappings.

Although this particular guideline was not explicitly tested in our user study, we have already noted that there is 
evidence indicating how difficult it is for people to judge the S-R compatibility of a design [27, 29]. Our user study and results should demonstrate to designers that they need to be willing to look beyond their own intuition when building a good user interface. Even a visual element that is as familiar and simple as a pointing cursor can have a perceivable impact on how well an interface actually works.

The user study and results presented here merit future study and additional research. Although we did not find any specific S-R compatibility effects for movement precision, it remains unclear whether different directional cursors can influence movement precision. Future experiments will look at this particular aspect of S-R compatibility more carefully. In addition, because we believed it was important to minimize bias due to handedness, we chose to use a subject pool consisting only of right-handed participants. It would be interesting to see if any S-R compatibility effects are "mirrored" in an opposite fashion with left-handed users. These kinds of effects are especially important since there is relatively less research on left-handed users and there appears to be interest in looking at how the individual differences between users can influence performance.

Another study could consider evaluating different kinds of orientation-neutral cursor representations. In this study, we chose a circle representation, but crosshairs are frequently considered to be equally "neutral." It would be valuable to know if all supposedly orientation-neutral cursors yielded the same user pointing performance, or if other implicit visual cues in these cursors could influence the S-R compatibility of user input.

The circular cursor used in this study had no visible hotspot though subjects in our study appeared to understand that it was located at the center of the circle. In practice, designers might consider augmenting circular cursors with a dot in the center to visibly indicate the location of the hotspot. Cursor size was not studied in the current experiment, though future work could investigate the impact of overall cursor size on SR-compatibility effects and direction of movement.

With respect to pen input, we saw that S-R compatibility effects were less pronounced compared to the other evaluated input methods. One possible explanation for this could be that while devices such as the Tablet PC provide visual cursors, the seen hand provides more compelling visual (and proprioceptive) cues than the cursor does. A future experiment could look specifically at pen input and the S-R compatibility implications of using different kinds of cursor representations versus no cursor feedback at all.

Our results also open up the possibility of some interesting modifications to existing forms of visual feedback for pointing interactions in GUIs. In the future, GUIs may also be able to take into account the S-R compatibility characteristics for individual users, adaptively tailoring cursor representations and other visual elements to facilitate performance for each unique user. It may also be possible to incorporate a dynamic, predictive cursor based on the current direction of movement so that appropriate directional cues are always given to users at all times during interaction. Though such a scheme for pointing cursors has yet to be developed and extensively studied, such dynamic cursors are already commonplace to indicate changes in system state. A practical implementation would have to overcome problems such as predicting changes in movement direction and achieving a sufficiently high update rate to be useful. Based on the data collected in this experiment, a "perfectly dynamic" cursor could theoretically improve pointing performance by as much as five percent, although choice of input device, effective index of difficulty, and other considerations like attentional focus could influence this performance gain in practice.

\section{CONCLUSION}

We conducted a controlled evaluation of different cursor orientations to look for possible stimulus-response compatibility effects on movement performance. Our results indicate that cursors cued for movement in specific directions can influence performance and that orientationneutral cursors may be preferable in situations where avoidance of directional bias is preferred. Choice of appropriate cursor representations could be especially important for input devices that are more difficult to use, such as freehand pointers.

\section{ACKNOWLEDGMENTS}

This research was funded by the Natural Sciences and Engineering Research Council of Canada (NSERC). We thank the reviewers for their comments and suggestions, which have been valuable in the revision of this paper.

\section{REFERENCES}

1. Broadbent, D. E., and Gregory, M. Donders' b- and creactions and S-R compatibility. Journal of Experimental Psychology 63, APA (1962), 575-578.

2. Callahan, J., Hopkins, D., Weiser, M., and Shneiderman, B. An empirical comparison of pie vs. linear menus. Proc. CHI 1988, ACM Press (1988), 95-100.

3. Card, S. K., English, W. K., and Burr, B. J. Evaluation of mouse, rate-controlled isometric joystick, step keys, and text keys for text selection on a CRT. Ergonomics 21, Taylor \& Francis (1978), 601-613.

4. Card, S. K., Mackinlay, J. D., and Robertson, G. G. The design space of input devices. Multimedia Interface Design, ACM Press (1992), 217-232.

5. Cho, Y. S., and Proctor, R. W. Effect of an initiating action on the up-right/down-left advantage for vertically arrayed stimuli and horizontally arrayed responses. Journal of Experimental Psychology: HPP 27(2), APA (2002), 472-484.

6. Cho, Y. S., and Proctor, R. W. Influences of hand posture and hand position on compatibility effects for 
up-down stimuli mapped to left-right responses: Evidence for a hand referent hypothesis. Perception and Psychophysics 64(8), Psychonomic Society (2002), 1301-1315.

7. Chua, R., Weeks, D. J., Ricker, K. L., and Poon, P. Influence of operator orientation on relative organizational mapping and spatial compatibility. Ergonomics 44(8), Taylor \& Francis (2001), 751-765.

8. Citrin, W., Halbert, D., Hewitt, C., Meyrowitz, N., and Shneiderman, B. Potentials and limitations of pen-based computers. Proc. CSC 1993, ACM Press (1993), 536539.

9. Fitts, P. M., and Seeger, C. M. S-R compatibility: Spatial characteristics of stimulus and response codes. Journal of Experimental Psychology 46, APA (1954), pp. 199-210.

10.Friesen, C., Ristic, J., and Kingstone, A. Attentional effects of counterpredictive gaze and arrow cues. Journal of Experimental Psychology: HPP 30(2), APA (2004), 319-329.

11.Hancock, M. S., and Booth, K. S. Improving menu placement strategies for pen input. Proceedings of Graphics Interface, 2004, pp. 221-230.

12.Hinckley, K., Pausch, R., Goble, J. C., and Kassell, N. F. A survey of design issues in spatial input. Proc. UIST 1994, ACM Press (1994), 213-222.

13. John, B. E., and Newell, A. Cumulating the science of HCI: From S-R compatibility to transcription typing. Proc. CHI 1989, ACM Press (1989), 109-114.

14.John, B. E., Rosenbloom, P. S., and Newell, A. A theory of stimulus-response compatibility applied to human-computer interaction. Proc. CHI 1985, ACM Press (1985), 213-219.

15. Kantowitz, B.H., Triggs, T. J., and Barnes, V. Stimulusresponse compatibility and human factors. In R.W. Proctor and T. Reeves (Eds.), Stimulus-Response Compatibility, Amsterdam: North Holland, 365-388.

16. Kirstein, C. and Mueller, H. Interaction with a projection screen using a camera-tracked laser pointer. Proc. Multimedia Modeling, (1998), 191-192.

17.Lippa, Y., and Adam, J. J. An explanation of orthogonal S-R compatibility effects that vary with hand or response position: The end-state comfort hypothesis. Perception and Psychophysics 63(1), Psychonomic Society (2001), 156-174.

18. MacKenzie, I. S. Fitts' law as a research and design tool in human-computer interaction. Human-Computer Interaction 7, (1992), 91-139.

19. MacKenzie, I. S., Sellen, A., and Buxton, W. A comparison of input devices in elemental pointing and dragging tasks. Proc. CHI 1991, ACM Press (1991), 161-166.
20. Myers, B. A., Bhatnagar, R., Nichols, J., Peck, C. H., Kong, D., Miller, R., and Long, A. C. Interacting at a distance: Measuring the effects of laser pointers and other devices. Proc. CHI 2002, ACM Press (2002), 3340.

21. Olsen, D. R., and Nielsen, T. Laser pointer interaction. Proc. CHI 2001, ACM Press (2001), 17-22.

22.Phillips, J. G., Meehan, J. W., and Triggs, T. J. Effects of cursor orientation and required precision on positioning movements on computer screens. International Journal of HCI 15(3), Lawrence Erlbaum (2003), 379-389.

23. Po, B. A., Fisher, B. D., and Booth, K. S. Mouse and touchscreen selection in the upper and lower visual fields. Proc. CHI 2004, ACM Press (2004), 359-366.

24. Ren, X. and Moriya, S. Improving selection performance on pen-based systems: A study of penbased interaction for selection tasks. ACM TOCHI 7(3), (2000), 384-416.

25. Sears, A. and Shneiderman, B. High precision touchscreens: Design strategies and comparisons with a mouse. International Journal of Man-Machine Studies 34(4), (1991), 593-613.

26. Shneiderman, B. Designing the User Interface: Strategies for Effective Human-Computer Interaction, Boston: Addison-Wesley (1992).

27. Tlauka, M. Display-control compatibility: The relationship between performance and judgments of performance. Ergonomics 47(3), Taylor \& Francis (2004), 281-295.

28. Vogt, F., Wong, J., Po, B. A., Argue, R., Fels, S. S., and Booth, K. S. Exploring collaboration with group pointer interaction. Proc. Computer Graphics International, (2004), 636-639.

29. Vu, K-P. L., and Proctor, R. W. Naïve and experienced judgments of stimulus-response compatibility: Implications for interface design. Ergonomics 46(1), Taylor \& Francis (2003), 169-187.

30. Worringham, C. J., and Beringer, D. B. Directional stimulus-response compatibility: A test of three alternative principles. Ergonomics 41(6), Taylor \& Francis (1998), 864-880.

31. Worringham, C. J., and Beringer, D. B. Operator orientation and compatibility in visual-motor task performance. Ergonomics 32(1), Taylor \& Francis (1989), 387-399.

32. Wulf, G. and Lee, T. D. Contextual interference effects in movements of the same class: Differential effects on program and parameter learning. Journal of Motor Behavior 25, (1993), 254-263. 